\title{
COMMENT
}

\section{Social media for pediatric research: what, who, why, and \#?}

\author{
Nicolas A. Bamat ${ }^{1}$, Brett J. Manley ${ }^{2}$, Matthew W. Harer $^{3}$ and Damian Roland ${ }^{4}$ \\ Pediatric Research (2018) 84:597-599; https://doi.org/10.1038/s41390-018-0140-7
}

In the age of Facebook congressional hearings and "Twitter diplomacy," the impact of social media on society is difficult to ignore. Although social media isn't new and its role in health care continues to grow, misconceptions of its purpose and utility in medicine are common, and some remain skeptical of its value. ${ }^{1}$ We provide a brief overview of the potential for social media to advance pediatric research and describe the use of hashtags, elaborating with an example from the neonatal clinical research community.

\section{WHAT IS SOCIAL MEDIA?}

The borders of social media are difficult to define. Broadly, we refer to internet-based technologies that facilitate the creation and sharing of digital information among networks of users. Facebook ( 2.2 billion active users) and Twitter (330 million active users) are popular platforms, others specifically focus on professional (Linkedln), academic (ResearchGate) or medical (Doximity) networks. ${ }^{2}$ Like all media, social media are a tool for communicating information. Specific features set them apart. First, they are digital, mobile-based technologies. Information is delivered to consumers through common devices like computers, tablets, and smartphones. A second distinguishing feature is the directionality of information. Unidirectional content flow from producer to consumer is typical of traditional media. Bidirectional flow within a complex network of nodes, each established by a user capable of both producing and consuming information, is typical of social media.

\section{WHO IS USING SOCIAL MEDIA?}

The use of social media to obtain information is increasing. Twothirds of adult Americans use social media to obtain news. ${ }^{3}$ Although the proportion of pediatric providers using social media professionally is unknown, its progressive adoption by the organizations and institutions performing, publishing, and funding pediatric research likely reflects a growing consumer base.

All free-standing United States children's hospitals, most journals publishing peer-reviewed pediatric research and each of the top 10 funders of health research in the world (Table 1) maintain active social media accounts. ${ }^{4,5}$

\section{WHY USE SOCIAL MEDIA USE IN MEDICAL RESEARCH?}

Social media use in the medical research community is motivated by a variety of challenges and incentives. We elaborate on three: knowledge dissemination, academic exposure, and collaborative networking.
Social media as a tool for knowledge dissemination Knowledge translation is needed for society to benefit from research. Ideally, valid research insights are quickly and widely disseminated to health care providers. Providers implement these insights as evidence-based practice changes, thereby improving outcomes identified as important by patients and their families. The reality is far from this ideal. A frequently cited 2001 Institutes of Medicine report concludes that health care routinely fails to deliver the potential benefits of research, preferring the concept of "chasm" over "gap" to describe the space between evidence and practice. Among the indictments is the 17-year average time required for information from randomized trials to reach practice. ${ }^{6}$ These shortcomings have motivated the emergence of "implementation science," a field focused on "methods to promote the systematic uptake of research findings and other evidence-based practices into routine practice," thereby improving health-care quality and effectiveness. ${ }^{7}$ Broadly, implementation science can be divided into two contiguous parts: (i) dissemination research, focused on the processes for distributing information to target audiences, and (ii) implementation research, focused on the processes for adopting and integrating that information into clinical settings. ${ }^{8}$

The evidence-practice chasm has been caused by various factors. Among them is the pace at which new knowledge requiring dissemination is generated. The global output of academic articles has doubled every nine years and reached 2.5 million articles in 2017. ${ }^{9}$ Remaining "up to date" is an increasingly daunting challenge for clinicians. Social media may help. Digital content delivery through omnipresent devices may help facilitate efficient, broad dissemination of information. In its Resident 360 series, the New England Journal of Medicine Group states that "checking the trending research studies on Twitter is one way to identify the most important published research each week. ${ }^{10 "}$ The International Society for Evidence-Based Neonatology (EBNeo) is one example of a medical society deliberately using social media for evidence dissemination. The non-profit group continuously reviews newly published neonatal research and highlights important findings through various social media platforms. ${ }^{11}$

Social media as a tool for academic exposure

Social media provides opportunities for funders, publishers and researchers to broaden their conventional exposure. Acknowledgment of research funding is commonly required from both public and philanthropic organizations. ${ }^{12,13}$ Public research funders like the United States National Institutes of Health state that such exposure "improves public understanding of how we, the biomedical research community as a whole, are working to improve

\footnotetext{
${ }^{1}$ Division of Neonatology and Center for Pediatric Clinical Effectiveness, The Children's Hospital of Philadelphia, Perelman School of Medicine at the University of Pennsylvania,

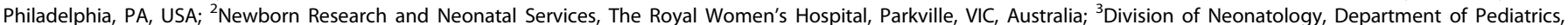
University of Wisconsin-Madison School of Medicine and Public Health, Madison, WI, USA and ${ }^{4}$ SAPPHIRE Group, Health Sciences, Leicester University, Leicester, UK Correspondence: Nicolas A. Bamat (bamatn@email.chop.edu)
}

Received: 9 July 2018 Accepted: 27 July 2018

Published online: 14 August 2018 
Table 1. Twitter handles (usernames) of the top 10 health research funders in the world (2012-13)

\begin{tabular}{ll}
\hline Research funder & Twitter handle \\
\hline National Institutes of Health (USA); public & @NIH \\
European Commission (EU); public & @EU_Commission \\
Medical Research Council (UK); public & @The_MRC \\
Institut national de la santé et de la recherche & @Inserm \\
médicale (FRA); public & \\
Department of Defense (USA); public & $@$ DeptofDefense \\
Wellcome Trust (UK); philanthropic & $@ w e l l c o m e t r u s t$ \\
$\begin{array}{l}\text { Canadian Institutes of Health Research (CAN); } \\
\text { public }\end{array}$ & @CIHR_IRSC \\
$\begin{array}{l}\text { National Health and Medical Research Council } \\
\text { (AUS); public }\end{array}$ & @nhmrc \\
Howard Hughes Medical Institutes (USA); & @hhminews \\
philanthropic & \\
Deutsche Forschungsgemeinschaft (GER); public & @dfg_public \\
\hline
\end{tabular}

human health." Further, positive exposure may increase public support for funding. The United Kingdom's National Institute for Health Research recently highlighted improved social media engagement as a goal of its five-year communications strategy. ${ }^{14}$

Medical publishers actively promote social media use. Share buttons exporting digital content to social media platforms are often displayed prominently on journal websites. Some journals have designated specific editors to implement social media strategies. ${ }^{15-19}$ One motivation may be to recruit high-quality content by demonstrating capacity for effective dissemination. ${ }^{20}$ Additional motivations may be increased brand recognition and reaching a broader consumer base. Social media posts routinely contain hyperlinks to journal websites, increasing digital traffic and providing increased revenue opportunities from new subscriptions and advertising. ${ }^{21}$

Individual researchers are increasingly incentivized to leverage social media for academic exposure. Sharing your research findings and opinions on social media allows a global audience to become familiar with your academic interests and perspectives. Altmetrics are non-traditional metrics proposed to complement traditional measures of academic success like the impact factor by measuring online digital activity around publications. ${ }^{22}$ An emerging literature suggests an association between altmetrics and traditional impact measures, albeit of unclear strength or causative nature. ${ }^{23}$ Companies such as Altmetric and Plum Analytics offer a quantitative summary of these measures, which are routinely displayed by medical journals. ${ }^{9}$ Some academic institutions have developed guidelines for incorporating social media metrics when recommending academic promotion. ${ }^{24}$

Social media as a tool for networking and collaborative research Professional networking is an often cited reason for adopting social media in academic medicine. ${ }^{18,25-27}$ The bidirectional flow of information between individuals and organizations with shared interests facilitates networking previously challenged by time, distance and a lack of mutual awareness. For example, the Collaboration for Outcomes on Social Media in Oncology (COSMO) "started working together after meeting online through Twitter, brought together by shared interests. ${ }^{25}$ " Further, social media may be an effective tool for facilitating collaborative research at scale. Early examples, such as multi-platform social media recruitment for multicenter surgical cohort studies, are being reported. ${ }^{28}$ Detecting small but meaningful treatment effects often requires data collection or subject enrollment at sample sizes that are not feasible for single centers or even traditional research networks. By facilitating communication and collaboration at global scale, social media may prove to be both a means to bridge the evidence-practice gap and a key engineer in generating new scientific insights.

\section{THE ROLE OF HASHTAGS IN SOCIAL MEDIA COMMUNICATION} Hashtags are words, acronyms or alphanumeric strings preceded by the pound (\#) sign. In social media, hashtags are often used to classify information on the basis of key content or themes - labels that allow information to be sorted into digital filing cabinets. Approximately 500 million tweets are generated each day; hashtags act as an informal, dynamic organization system to help users identify specific relevant content. ${ }^{29}$ Collaborative communities of practice have evolved around hashtags. ${ }^{30} \mathrm{~A}$ recent case from the neonatal research community provides an illustrative example. In the days prior to the Pediatric Academic Societies (PAS) 2018 meeting, a discussion on the use of hashtags surfaced among neonatal providers on Twitter. \#FOAMneo, an extension of \#FOAMed (free open-access medical education) was acknowledged as the most commonly used hashtag for labeling content targeted to the academic neonatal community. ${ }^{31}$ However, \#FOAMneo content was often not open-access, but restricted behind publisher paywalls. Following social media discussion of possible solutions, EBNeo used Twitter to organize an in-person meet-up at PAS. Acknowledging the importance of both promoting and distinguishing open-access content, those present agreed to retain \#FOAMneo to specify open-access content, while the hashtag \#NeoEBM was introduced to more broadly classify information related to neonatal evidence-based practice. In the seven weeks following its inception, \#NeoEBM appeared in 1925 posts from 502 user-accounts, garnering 2.5 million impressions on Twitter alone.

\section{CHALLENGES AND LIMITATIONS}

The use of social media to promote pediatric research faces various challenges and limitations:

By either tendency or design (Twitter), information is often shared in concise digital bites. This can facilitate confusion and misunderstanding. Scientific findings are sometimes complex, opinions and judgements sometimes nuanced-neither are well suited to restricted expression.

Numerous factors threaten the quality of information shared. The energy required to share information is minimal; citations are uncommon; peer review occurs post hoc. Opinion and fact blur readily, even when misinformation is not the intent. The challenges imposed by "fake news" in politics provide an example of the possible harms when misinformation is the intent. While the evidence-practice gap needs to be narrowed, changing practice too quickly-for example, on the basis of low-quality information-can cause harm.

Over-incentivizing exposure may have adverse effects. Academics may unwittingly stray from necessary to excessive self-promotion. Funders and publishers eager to drive traffic to websites may be biased towards the kind of sensationalism that engenders distrust. Lastly, a sad irony of social media is that these technologies meant to ease dialogue have also made it easier to ignore views that differ from our own. Social media software algorithms have facilitated our inherent tendencies to prefer affirmation over disagreement, allowing engagement within digital echo chambers that reinforce our existing views. Listening to a rich diversity of opinion and perspective from the various stakeholders in pediatric research will be critical in leveraging social media to further the pediatric research agenda. 


\section{FURTHER RESEARCH IS NEEDED}

We highlight the potential of social media for promoting pediatric research; understanding the true value will require ongoing dedicated scholarship. Traditional study design frameworks should be applied and when appropriate, modified to the unique context of social media. ${ }^{32}$ How and to what extent are pediatric health care providers using social media to obtain professional information? Can social media dissemination increase awareness, knowledge, practice change and ultimately, improve health outcomes? Can social media facilitate efficient, large-scale collaborative research? Can hashtags such as \#NeoEBM create and sustain a community of practice? All are questions that can and should be addressed through rigorous research.

\section{ADDITIONAL INFORMATION}

Competing interests: Dr.(s) Bamat and Manley hold non-compensated positions on the board of The International Society for Evidence Based Neonatology. The remaining authors declare no competing interests.

Publisher's note: Springer Nature remains neutral with regard to jurisdictional claims in published maps and institutional affiliations.

\section{REFERENCES}

1. Roland, D. Social media, health policy, and knowledge translation. J. Am. Coll. Radiol. 15, 149-152 (2018).

2. Statista. Most famous social network sites 2018 , by active users. https://www. statista.com/statistics/272014/global-social-networks-ranked-by-number-ofusers/. Accessed 28 June 2018

3. Shearer, E. \& Gottfried, J. News use across social media platforms 2017. Pew Research Center, Journalism and Media. http://www.journalism.org/2017/09/07/ news-use-across-social-media-platforms-2017/. Accessed 13 June 2018.

4. Viergever, R. F. \& Hendriks, T. C. C. The 10 largest public and philanthropic funders of health research in the world: what they fund and how they distribute their funds. Heal. Res. Policy Syst. 14, 12 (2016).

5. Wong, C. A. et al. How U.S. children's hospitals use social media: a mixed methods study. Heal J. Deliv. Sci. Innov. 4, 15-21 (2016).

6. Institute of Medicine (US) Committee on Quality of Health Care in America. Crossing the Quality Chasm: A New Health System for the 21st Century (National Academies Press, Washington, 2001).

7. Eccles, M. P. \& Mittman, B. S. Welcome to implementation science. Implement. Sci. 1, 1 (2006).

8. Neta, G., Brownson, R. C. \& Chambers, D. A. Opportunities for epidemiologists in implementation science: a primer. Am. J. Epidemiol. 187, 899-910 (2018).

9. Warren, H. R., Raison, N. \& Dasgupta, P. The rise of altmetrics. JAMA 317, 131-132 (2017).

10. Berger, R. \& Ramaswami, R. Keeping up with the medical literature. NEJM Resident 360. https://resident360.nejm.org/content_items/keeping-up-with-themedicalliterature/. Accessed 14 June 2018.
11. Evidence-based neonatology. About. https://ebneo.org/about/. Accessed 17 June 2018.

12. Wellcome. Research publication acknowledgement: requirement for authors. https:// wellcome.ac.uk/funding/managing-grant/research-publicationacknowledgementpractice-guidance-authors. Accessed 14 June 2018.

13. National Institutes of Health. Office of Extramural Research. Communicating and acknowledging federal funding. https://grants.nih.gov/grants/acknow.htm. Accessed 14 June 2018.

14. National Institutes for Health Research. NIHR communications strategy (2017-2022). https://www.nihr.ac.uk/aboutus/documents/NIHR\%20communications\%20strategy. pdf. Accessed 7 July 2018

15. Cippà, P. E. Social media editor: what is it all about? Transpl. Int. 29, 390-391 (2016).

16. Ramasamy, R. Social Media Editor: Dr. Ranjith Ramasamy. Urology 90, 2 (2016).

17. Olson, C. A. JCEHP's new-and first-Social Media Editor. J. Contin. Educ. Health Prof. 34, 199 (2014).

18. Niehaus, W. N., Silver, J. K. \& Katz, M. S. The PM\&R journal implements a social media strategy to disseminate research and track alternative metrics in physical medicine and rehabilitation. PM\&R. 10, 538-543 (2018).

19. Pediatric Research. Announcement: Pediatric Research Social Media Editor-Dr. Damian Roland. https://www.nature.com/pr/. Accessed 7 July 2018.

20. JAMA Network. JAMA pediatrics. For authors. Why publish in JAMA pediatrics? https://jamanetwork.com/journals/jamapediatrics/pages/for-authors\#fawhypublish. Accessed 14 June 2018.

21. Fox, C. S., Barry, K. \& Colbert, J. Importance of social media alongside traditional medical publications. Circulation 133, 1978-1983 (2016).

22. Priem, J., Groth, P. \& Taraborelli, D. The altmetrics collection. PLoS ONE 7, e48753 (2012).

23. Thelwall, M., Haustein, S., Larivière, V. \& Sugimoto, C. R. Do altmetrics work? Twitter and ten other social web services. PLOS ONE 8, e64841 (2013).

24. Cabrera, D. et al. More than likes and tweets: creating social media portfolios for academic promotion and tenure. J. Grad. Med. Educ. 9, 421-425 (2017).

25. Attai, D. J. et al. Risks and benefits of Twitter use by hematologists/oncologists in the era of digital medicine. Semin. Hematol. 54, 198204 (2017).

26. Mayol, J. \& Dziakova, J. Value of social media in advancing surgical research. Br. J. Surg. 104, 1753-1755 (2017).

27. Choo, E. K. et al. Twitter as a tool for communication and knowledge exchange in academic medicine: a guide for skeptics and novices. Med. Teach. 37, 411-416 (2015).

28. Khatri, C. et al. Social media and internet driven study recruitment: evaluating a new model for promoting collaborator engagement and participation. PLOS ONE 10, e0118899 (2015).

29. Internet Live Stats. Twitter usage statistics. http://www.internetlivestats.com/ twitter-statistics/. Accessed 18 June 2018.

30. Roland, D., Spurr, J. \& Cabrera, D. Preliminary evidence for the emergence of a health care online community of practice: using a netnographic framework for Twitter hashtag analytics. J. Med. Internet Res. 19, e252 (2017).

31. Nickson, C. P. \& Cadogan, M. D. Free Open Access Medical education (FOAM) for the emergency physician. Emerg. Med. Australas. 26, 76-83 (2014).

32. Roland, D., Spurr, J. \& Cabrera, D. Initial standardized framework for reporting social media analytics in emergency care research. West J. Emerg. Med. Integr. Emerg. Care Popul. Heal. 19. https://escholarship.org/uc/item/9xw166w4. Accessed 7 July 2018 (2018). 